\section{Preventive and conservative prosthodontic treatment using overdenture and richmond crown}

\author{
Jevin S. Cialy," Bahruddin Thalib, Mohamad Dharmautama, \\ Wahipa Wiro, Ervina
}

CrossMark

\title{
Abstract
}

Objective: Prosthodontic treatment aims to restore the chewing function (rehabilitation) and to prevent tooth decay and bone resorption (preventive and conservative). By retaining the original teeth and/or roots, it could improve the stability and retention of the denture and defend proprioseptic of teeth and vertical dimensions. The aim of this study is to provide information about preventive prosthodontic treatment and prevention by using overdenture and Richmond crown.

Methods: A 50 year old woman only has 44 teeth on the mandible and loss teeth 13, 14, 17 and 27 on the maxilla with deep caries in teeth 22 .
On an early stage study, printing models is done. Then treatment of the root canal on teeth 22 and 44 , followed by root canal preparation, core construction and Richmond crown on tooth 22 and bareroot preparation on tooth 44 for overdenture construction.

Results: Using over denture has proved better than using of full denture because it can minimize the occurrence of alveolar bone resorption.

Conclusion: Choosing treatment either overdenture or richmond crown is very important because it could mantain remain original teeth, thus it can help to improve the retention and stabilization.
Department of Prostodontic, Faculty of Dentistry, Hasanuddin University, Makassar, Indonesia

*Correspondence to:

Jevin Stivie Cialy,

jevin_s_c@yahoo.com

Received: 03 0ctober 2016

Revised: 15 November 2016

Accepted: 22 November 2016

Available online:18 December 2016

Keywords: Overdenture, Prosthodontic treatment, Richmond

Cite This Article: Cialy JS, Thalib B, Dharmautama M, Wiro W, Ervina. 2016. Preventive and conservative prosthodontic treatment using overdenture and richmond crown. Journal of Dentomaxillofacial Science 1(3): 193-195. D0I: 10.15562/jdmfs.v1i3.316

\section{Intoduction}

Prosthetics is a branch of dentistry that includes restoration and treatment of oral function by replacing teeth and missing structures with a replacement tooth or denture. Prosthetic care should be focus on prevention procedures of excessive alveolar bone loss and can maintain the shape of the ridge alveolar. ${ }^{1,2}$

Conservative and preventive prosthetic treatment is a form of denture treatment preventing potential prosthetics post-treatment issues. In patients with few teeth remaining and had erupted, repealing should be planned before considering the advantages of retaining remaining tooth roots and alveolar bone area. Revocation of all teeth and replaced with a complete denture is not a proper treatment, especially in the mandible. Extracted human teeth were subsequently replaced with complete dentures can cause various complications, such as progressive alveolar bone resorption due to the effect of occlusion pressure from the denture to the mucosa, as well as denture could not be last long and may cause neuromuscular pain to patients. The use of retention on one or more of the tooth root for overdenture and crown richmond treatment plan may provide advantages over functionally and biologically in the oral cavity of patients. ${ }^{2}$

Overdenture is a removable partial or complete denture that covers and leans on one or more of the original tooth, tooth roots and/or dental implants. Overdenture nowadays often used for treatment of periodontics, endodontics and caries control technique. By maintaining the element of original teeth and/or roots, increases stability and retention of the denture, as well as maintains the sensory stimuli and vertical dimensions. Overdenture is a treatment that can prevent alveolar bone resorption, provide better load transmission, keep the sensory feedback and achieve good denture stability. Retention and stability of overdenture may be improved by using original teeth, precision connection or magnet. ${ }^{2,3}$

Richmond crown is fixed denture with post and crown (single unit) systems, where the post part goes into the root canal and its crown cover the entire of the original tooth crown surface. Indications for richmond crown is not a lot, usually is done on a tooth which has been badly damaged so that the remained tooth structure is very little, or in the case of incisal inclination repair (deep bite and too small overjet). Due to the tooth structure in the cervical when the load gets to be functioned, this design protects the cervical part of post-core system than the others, so as protects the tooth crown margin and reduce the possibility of restoration leakage. ${ }^{4,5}$

In this paper reported a case of using the richmond crown and overdenture by maintaining 
the roots of original teeth so it can develop preventative and conservative prosthodontic treatment.

\section{Case Report}

A 50 year old woman presented to the Dental Hospital Hasanuddin University after experiencing difficulty of chewing food and perforated front teeth. Patient's general condition is good and no systemic disorders. By intraoral examination, there are deep carious tooth 22 and tooth loss on $13,14,17$ and 27 in the maxilla and only 44 teeth on the mandible figure 1. Radiographic examination showed no presence of radiolucent on teeth 22 and 44, deep
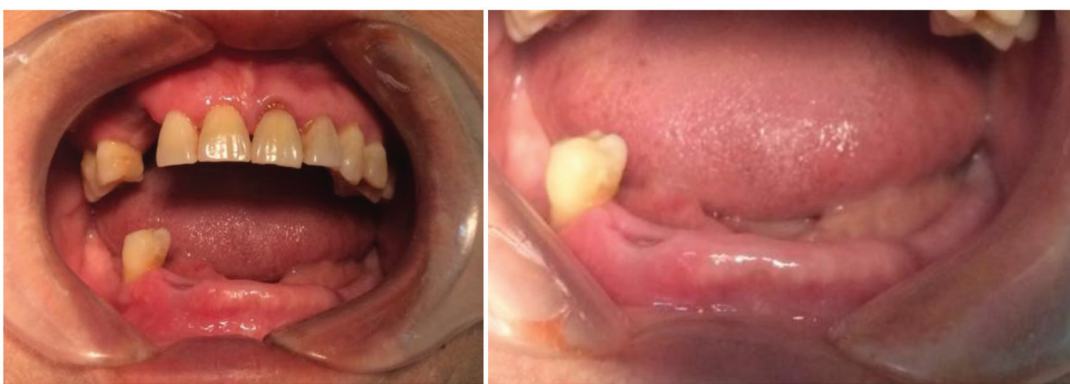

Figure 1 Maxillary and mandibular intraoral before denture construction
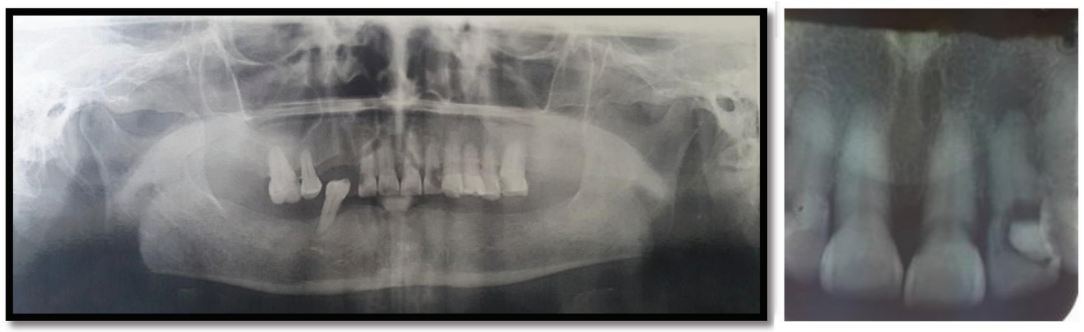

Figure 2 RO before denture construction

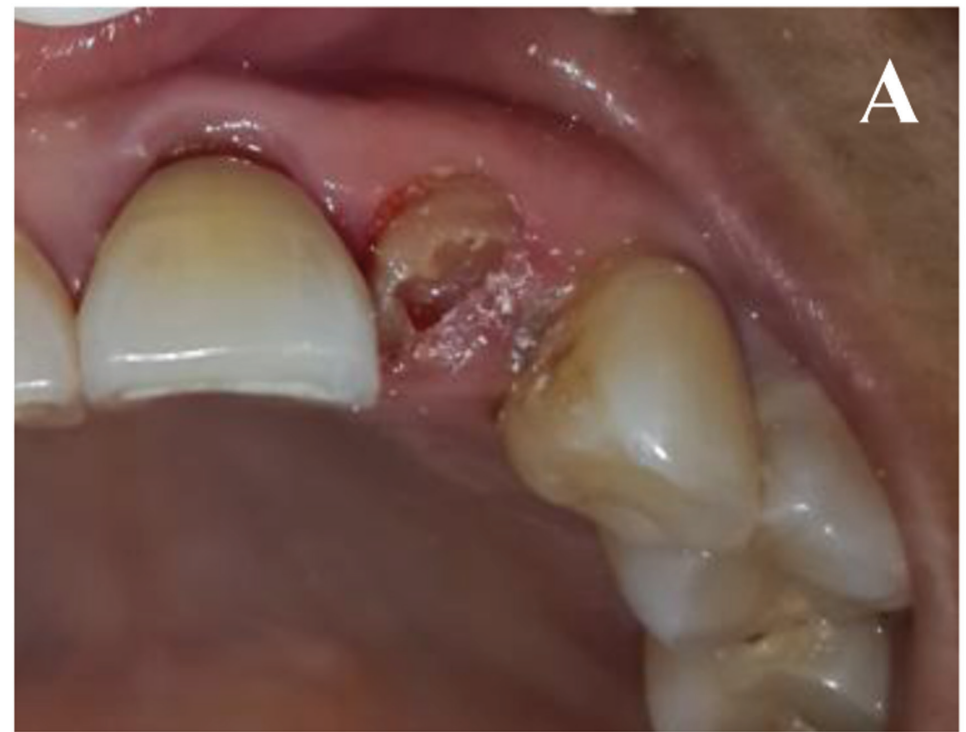

caries in teeth 22 and the ratio of crown roots of 1: 2 figure 2 .

The treatment which was carried out in this case is overdenture construction for tooth 44 on the mandible and removable partial denture (RPDs) with richmond crown gear 22 on the maxilla. At early stage, printing for diagnostic model creation and construction of temporary denture. Then treated on canal tooth root 22 and 44, followed by root canal and ferrule preparation for the construction of richmond crown gear 22 and the barerootpreparation on teeth 44 for the construction of overdenture. Figure 3 The procedure followed by physilogical printing double impression of the maxilla and the mandible was done separately to get a working model, then recorded the bite. Try in the post on tooth 22 and try in anterior and posterior teeth on the mandible, then the determination of color using vitapan shade guide, then proceed with the lab of Richmond crown and overdenture which have completed at insertion figure 4 .

\section{Discussion}

Loss of teeth can cause loss of periodontal ligament and tissue around it which influencing alveolar bone and raising alveolar bone resorption. Alveolar bone cannot survive to get large pressure from full denture because of using full denture can cause alveolar bone resorption constantly and rapidly. Using full denture potent to cause alveolar bone resorption, in which alveolar bone resorption in mandible is larger than that in maxilla. Process of alveolar bone resorption due to the use of full denture can cause the decreasing of residual ridge and this is progressive and irreversible chronic disease. It is suitable according to the invention

Figure 3 A. Preparation of the root canal and ferrule tooth 22, B. Preparation bare root of tooth 22 

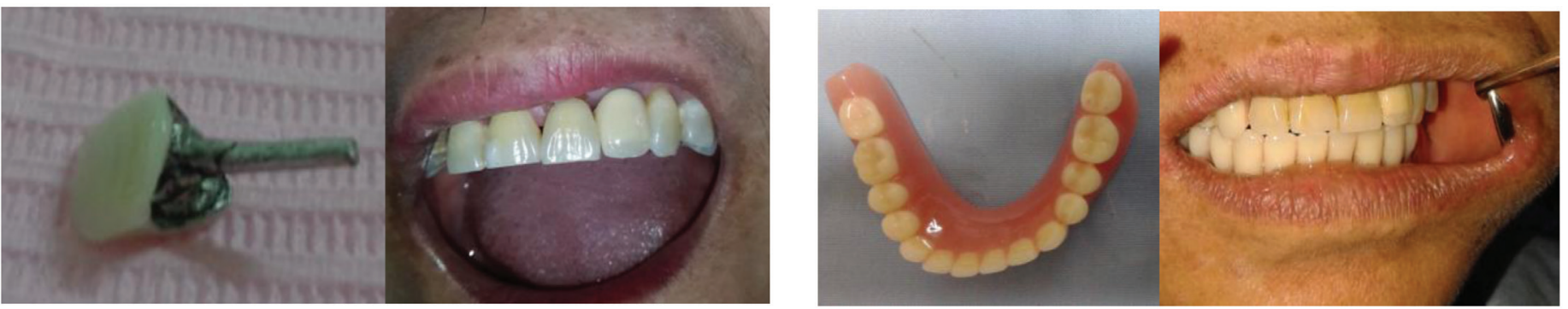

Figure 4 A. Insertion of Richmond crown gear 22, B. Insertion of Overdenture RB

of Kanneganti et al. ${ }^{2}$ who had proven that patient with lower anterior teeth, when applied to the full maxilla denture, it visibly causes a decreasing in the residual ridge in the anterior maxilla. Using full denture continuously can improve the decreasing of the residual ridge. The occurrence of resorption and remodeling of alveolar bone causing retention, stabilization and support the denture are not optimal. ${ }^{1,2}$

Using over denture has proved better than using of full denture because it can minimize the occurrence of alveolar bone resorption. According to Soesetijo ${ }^{3}$ using of overdenture can protect alveolar bone by utilizing of remaining tooth root as a buffer and protects the bones and also maintain alveolar bone height. Using of overdenture can produce retention and stabilization of denture as well because of alveolar bone height is still awake so that the pressure of mastication in mucous is reduced because of vertical pedestal of root abutment. Soesetijo ${ }^{3}$ reported that overdenture patient have ability to digest three times larger than full denture patient. The advantages of using overdenture in this case are low cost treatment, repeated treatment, maintaining prospective stimulus and preventing bone resorption. ${ }^{3}$

Using richmond crown can minimize the occurrence of alveolar bone resorption as well. Richmond crown is a fixed denture with post system and crown (single unit), which is the post enter into the root canal and it covers the entire surface of original tooth crown. Abhinav ${ }^{4}$ said that successful treatment on teeth that have suffered severe damage not only depends on good endodontic therapy but also good prosthetic reconstruction and protecting of bone resorption. In this case, richmond crown is better treatment options than prefabricated posts because it can protect cervical section of teeth, reduce the occurrence of leak restoration especially when functional pressure have occurred and maintain proprioceptive stimulus. ${ }^{4,5}$

\section{Conclusion}

Overdenture and richmond crown, are so important because can maintain remaining natural teeth, maintain proprioceptive stimulus and protect bone resorption, thus they improve denture retention and stabilization.

\section{References}

1. Bakar A. Kedokteran gigi klinis. Yogjakarta: Quantum Sinergis Media; 2012. p. 147.

2. Kanneganti KC, Abbasi M, Reddy VV, et al. Preventive prosthodontics by precision attachment- a case report. Sch J Dent Sci 2016;3: 45-50.

3. Soesetijo FXA. Overdenture: perawatan dengan pendekatan preventif dan konservatif. CDK-190 2012;39: 102-103.

4. Abhinav A, Manish C, Kumar DRV, et al. Richmond crown-a conventional approach for restoration of badly broken posterior teeth. J Dent Peer 2013;1: 35-39.

5. Rupika G, Jagadish S, Shashikala K, et al. Restoration of badely broken, endodontically treated posterior teeth. J Conserv Dent 2009;12: 123-128.

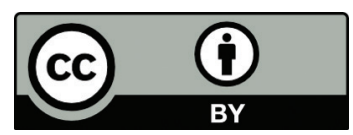

This work is licensed under a Creative Commons Attribution 